\title{
Kruppel-like factor 15 is critical for vascular inflammation
}

\author{
Yuan Lu, ${ }^{1}$ Lisheng Zhang, ${ }^{2}$ Xudong Liao, ${ }^{1}$ Panjamaporn Sangwung, \\ Domenick A. Prosdocimo, ${ }^{1}$ Guangjin Zhou, ${ }^{1}$ Alexander R. Votruba, ${ }^{1}$ Leigh Brian, ${ }^{2}$ \\ Yuh Jung Han, ${ }^{1}$ Huiyun Gao, ${ }^{1}$ Yunmei Wang, ${ }^{1}$ Koichi Shimizu, ${ }^{3}$ Kaitlyn Weinert-Stein, ${ }^{1}$ \\ Maria Khrestian, ${ }^{1}$ Daniel I. Simon, ${ }^{1}$ Neil J. Freedman, ${ }^{2}$ and Mukesh K. Jain ${ }^{1}$ \\ ${ }^{1}$ Case Cardiovascular Research Institute, Case Western Reserve University, and Harrington Heart and Vascular Institute, \\ University Hospitals Case Medical Center, Cleveland, Ohio, USA. ²Division of Cardiovascular Medicine, \\ Duke University Medical Center, Durham, North Carolina, USA. ${ }^{3}$ Division of Cardiovascular Medicine, \\ Brigham and Women's Hospital and Harvard Medical School, Boston, Massachusetts, USA.
}

\begin{abstract}
Activation of cells intrinsic to the vessel wall is central to the initiation and progression of vascular inflammation. As the dominant cellular constituent of the vessel wall, vascular smooth muscle cells (VSMCs) and their functions are critical determinants of vascular disease. While factors that regulate VSMC proliferation and migration have been identified, the endogenous regulators of VSMC proinflammatory activation remain incompletely defined. The Kruppel-like family of transcription factors (KLFs) are important regulators of inflammation. In this study, we identified Kruppel-like factor 15 (KLF15) as an essential regulator of VSMC proinflammatory activation. KLF15 levels were markedly reduced in human atherosclerotic tissues. Mice with systemic and smooth muscle-specific deficiency of KLF15 exhibited an aggressive inflammatory vasculopathy in two distinct models of vascular disease: orthotopic carotid artery transplantation and diet-induced atherosclerosis. We demonstrated that KLF15 alters the acetylation status and activity of the proinflammatory factor $\mathrm{NF}-\mathrm{KB}$ through direct interaction with the histone acetyltransferase $\mathrm{p300}$. These studies identify a previously unrecognized KLF15-dependent pathway that regulates VSMC proinflammatory activation.
\end{abstract}

\section{Introduction}

Inflammation is central to the pathogenesis of numerous vascular disease states that collectively serve as a major source of morbidity and mortality worldwide (1-4). A number of cell types, both intrinsic and extrinsic to the vessel wall, are activated by inflammatory stimuli. In turn, these cellular constituents perpetuate the inflammatory response, resulting in a vicious feedforward cycle that contributes to disease progression and its attendant complications such as heart attack, stroke, and gangrene of the extremities $(1,4-6)$. Despite the recognized importance of inflammation in disease states such as atherosclerosis or transplant vasculopathy, therapies directed at limiting inflammation are lacking $(1,4,7-11)$. As such, the identification of novel molecular pathways that limit inflammation is of considerable interest.

As the most abundant cell intrinsic to the vessel wall, the vascular smooth muscle cell (VSMC) is central to blood vessel homeostasis. Under physiologic conditions, this quiescent cell regulates vasomotor tone and blood pressure. However, in response to injury, VSMCs proliferate, migrate, secrete ECM, and elaborate cytokines/chemokines that promote and amplify inflammation $(1,7,8)$. While a number of factors have been implicated in the proproliferative/promigratory response $(9,10,12)$, our understanding of endogenous factors that regulate VSMC inflammatory activation remains poorly understood.

Conflict of interest: The authors have declared that no conflict of interest exists. Note regarding evaluation of this manuscript: Manuscripts authored by scientists associated with Duke University, The University of North Carolina at Chapel Hill, Duke-NUS, and the Sanford-Burnham Medical Research Institute are handled not by members of the editorial board but rather by the science editors, who consult with selected external editors and reviewers.

Citation for this article: J Clin Invest. 2013;123(10):4232-4241. doi:10.1172/JCI68552.
Studies over the past decade have implicated the Kruppel-like family of transcription factors (KLFs) as central regulators of blood vessel biology (13). With respect to VSMCs, three members of this gene family have been identified as regulators of this cell type in vivo, namely, KLF4, KLF5, and KLF15 (14). Although KLF4 and KLF5 are minimally expressed in VSMCs under basal conditions, they are rapidly induced upon injury and regulate smooth muscle differentiation and proliferation (14). By contrast, KLF15 is robustly expressed in VSMCs under basal conditions, but is reduced following mechanical injury or exposure to proproliferative/proinflammatory stimuli (14). However, a role for any VSMC-KLFs in inflammatory vascular disease remains unknown. Here, we show that KLF15 expression is significantly reduced in human atherosclerotic tissues $(P=0.0001)$. Further, SMC-specific deficiency of KLF15 renders vessels susceptible to increased inflammation. Mechanistically, we show that KLF15 alters the acetylation status and activity of NF- $\mathrm{\kappa B}$ through direct interaction with p300. Taken together, these studies identify KLF15 as a novel molecular regulator of vascular inflammation and elucidate a previously unrecognized molecular pathway that regulates VSMC proinflammatory activation.

\section{Results}

KLF15 expression is reduced in human atherosclerotic tissues. To explore the potential role of KLFs in atherogenesis, we first examined the expression profile of KLFs in human atherosclerotic aortic samples. In comparison with nonatherosclerotic control aortae $(n=6)$, KLF15 mRNA expression was significantly decreased by approximately 7 -fold in atherosclerotic aortic specimens $(n=6$, $P=0.0001)$ (Figure 1A). The expression of two additional KLF family members, KLF2 and KLF4, was also significantly decreased in atherosclerotic aortae by approximately 2 -fold $(P=0.02)$ and 
A

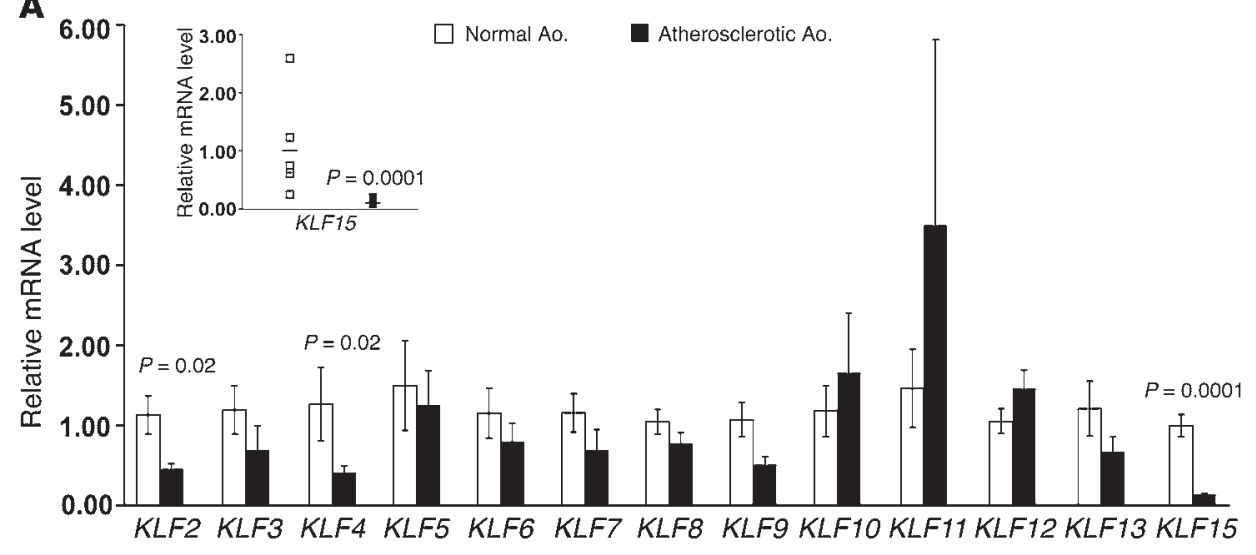

B

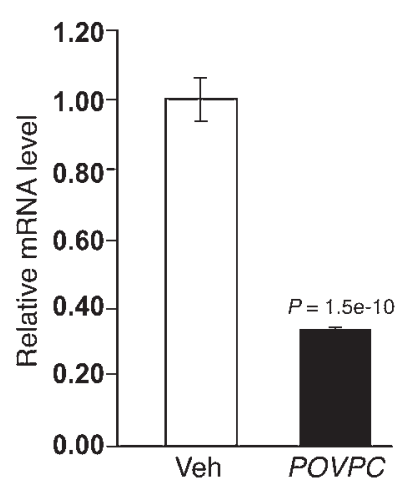

\section{Figure 1}

KLF15 is decreased in human atherosclerotic tissue. (A) KLF mRNA expression in human atherosclerotic tissue $(n=6)$. Values are normalized to $\beta$-actin and presented as the mean \pm SEM. Insert shows individual KLF15 expression data. (B) KLF15 mRNA expression in RASMC after POVPC $(100 \mu \mathrm{M})$ treatment for 12 hours $(n=12)$. Values are normalized to $18 \mathrm{~S}$ rRNA and presented as the mean \pm SD. Ao., aorta.

3-fold $(P=0.02)$, respectively (Figure $1 \mathrm{~A}$ and Supplemental Figure $1 \mathrm{~A}$; supplemental material available online with this article; doi:10.1172/JCI68552DS1). We next assessed Klf15 mRNA expression in primary rat aortic smooth muscle cells (RASMCs). Consistent with the in vivo observations, treatment of primary RASMCs with the oxidized phospholipid 1-palmitoyl-2-(5-oxovaleroyl)-sn-glycero-3-phosphocholine (POVPC), an active component of minimally modified LDL and a proatherogenic stimulus, significantly reduced Klf15 expression (Figure 1B). Since KLF15 expression is reduced in human atherosclerotic vascular tissues as well as in POVPC-challenged RASMCs, we postulated that vascular KLF15 may play an important role in atherogenesis.

Systemic KLF15 deficiency accelerates atherogenesis. We next sought to determine the effect of KLF15 deficiency on atherogenesis. As a first step, we examined the relative expression of KLF15 in vascular and myeloid cells. RNA was harvested from freshly isolated mouse aortic SMCs (MASMCs) (passage 2), cardiac microvascular endothelial cells (CECs) (passage 2), and both thioglycollate-elicted and bone marrow-derived macrophages (macrophage-P or macrophage-BM) (passage 1). As shown in Supplemental Figure 1B, Klf15 was expressed virtually exclusively in MASMCs. To confirm this in vivo, we used a $\mathrm{Klfl}^{+/-}$reporter mouse in which the KLF15 locus was targeted using a nuclear-localized LacZ cassette. Costaining was performed for endothelial (vWF) cells and SMCs (SMC $\alpha$-actin). As shown in Supplemental Figure 1C, Klf15 expression was limited to the medial SMC layer.

Next, to determine whether KLF15 deficiency affects atherogenesis, KLF15-null mice on an Apoe-/- background were challenged with a high-fat diet (HFD) for two time points (15 or 25 weeks), and the extent of aortic atherosclerotic plaques was assessed en face after Sudan IV staining. Aortae from Klf15 $5^{-/-} / A_{p o e^{-/}}$mice displayed a significantly greater plaque lesion size compared with control Apoe $e^{-/}$mice at both time points (Supplemental Figure 2,

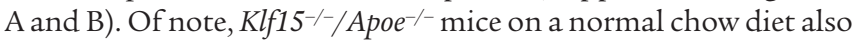
developed enhanced atherosclerosis after 25 weeks (Supplemental Figure 2, A and B). In addition, Klf15 $5^{-/} / \mathrm{Apoe}^{-/-}$mice demonstrated significantly higher mortality than $A p o e^{-/-}$mice in the HFD-fed groups: $20 \%$ versus $0 \%$ for 15 weeks, and $40 \%$ versus $9 \%$ for 25 weeks, respectively (data not shown). Klf15 $5^{-/-} /$Apoe $^{-/-}$mice also showed significantly higher levels of plasma total cholesterol and triglycerides (Supplemental Figure 2, C and D), findings that may contribute to the proatherosclerotic phenotype observed in KLF15-null animals.

SMC KLF15 deficiency promotes vascular inflammation. To eliminate the confounding effects of hyperlipidemia, we undertook two distinct approaches to assess the effect of SMC KLF15 deficiency on atherogenesis. First, we orthotopically transplanted carotid arteries from WT and congenic Klf15 $15^{-/}$mice into congenic Apoe-/- mice as we have described previously (15). Four weeks following transplantation, KLF15-deficient carotid grafts developed atherosclerotic lesions that were approximately 2 -fold larger than those that developed in WT carotid grafts (Figure 2, A and B). These larger Klf15 $15^{--}$carotid lesions had a $24 \% \pm 7 \%$ greater prevalence of macrophages $(n=6, P<0.001)$, but fewer VSMCs $(50 \% \pm 20 \%)$ than were observed in the WT carotid lesions (Figure $2 \mathrm{C}$ and data not

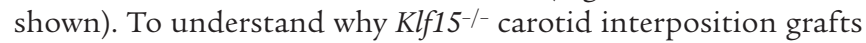
recruited more macrophages, we first isolated VSMCs from WT and KLF15-null aortae and tested protein expression levels of a panel of proinflammatory and proatherosclerotic targets by ELISA $(12,16-24)$. Of the 22 targets tested, 6 targets were significantly increased, including monocyte chemotactic protein 1 (MCP-1), vascular cell adhesion molecule 1 (VCAM-1), matrix metalloproteinase 3 (MMP3), PDGFAA, and PDGFAB (Figure 2D), while VEGF levels were significantly lower in KLF15-null VSMCs. To verify these observations in tissues, we harvested carotid grafts 1 week postoperatively, before intimal macrophage infiltration and immunostained for proteins responsible for recruiting and retaining monocytes/macrophages MCP-1 and VCAM-1 in the arterial intima, as described previously (15). Concordant with the greater recruitment of macrophages at 4 weeks after interposition grafting, preatherosclerotic $\mathrm{Klfl}^{-/-}$carotid grafts expressed $30 \%-40 \% \pm 10 \%$ more MCP- 1 and VCAM- 1 molecules than WT carotid grafts (Figure 2E). Thus, data from both carotid grafts in vivo and VSMCs in vitro demonstrated that KLF15 deficiency augments inflammatory signaling.

As a second approach, we used the Cre-LoxP system to study the endogenous role of VSMC KLF15 in atherogenesis. SMC-specific deletion was accomplished with the use of the $\operatorname{Sm} 22 \alpha-C r e$ 
A

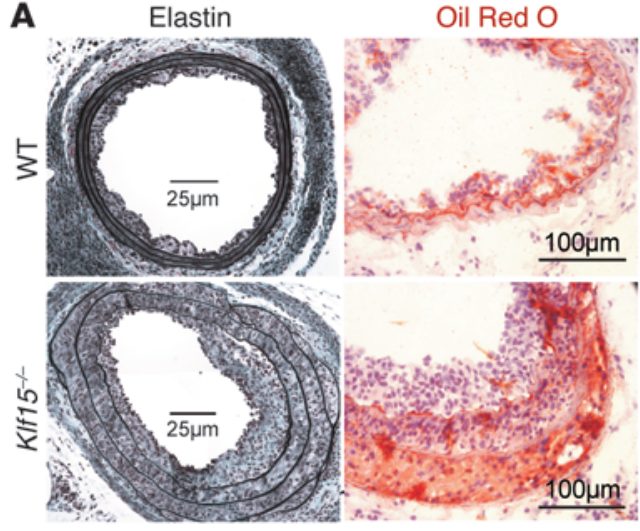

B Carotid graft cross-section (4 weeks after operation)

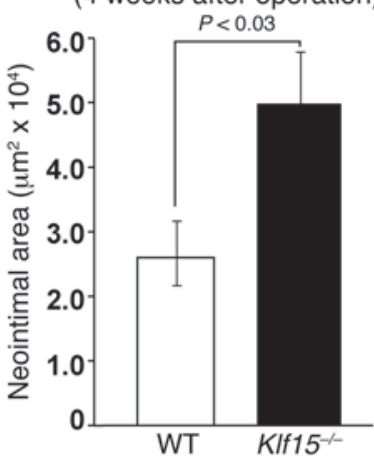

C
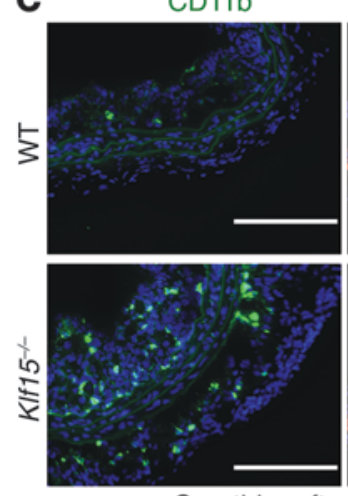

Carotid graft cross-section

(1 week after operation)
D

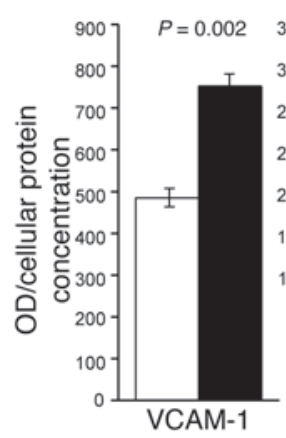

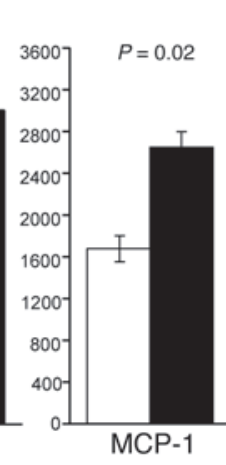
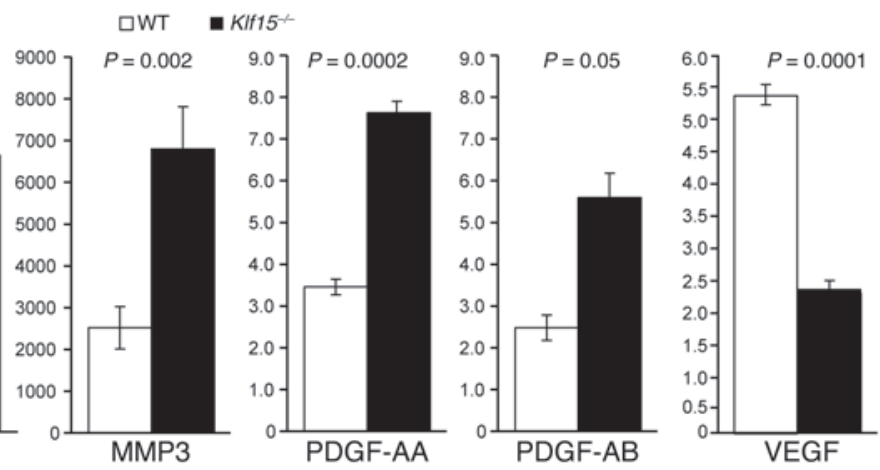

E

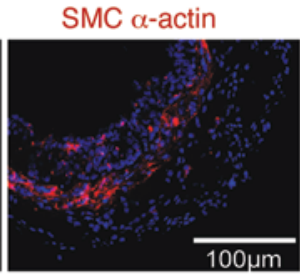

\section{Figure 2}

Arterial KLF15 expression reduces atherogenesis. (A and B) Carotid arteries from WT and Klf15 ${ }^{-/-}$mice were transplanted orthotopically into congenic $\mathrm{Apoe}^{-/-}$mice, harvested 4 weeks later, and stained with a connective tissue stain (Elastin) or a lipid stain (Oil Red O). Neointimal cross-sectional areas were measured by planimetry and plotted as the means \pm SEM of greater than or equal to 6 specimens in each group. (C) Serial sections of specimens from A were immunostained for monocytes/macrophages (CD11b) or VSMCs (SMC $\alpha$-actin). (D) Aortic VSMCs from WT and $\mathrm{KIf15}^{-/-}$mice were serum starved for 24 hours and then changed into DMEM medium with no serum for 6 hours. The conditioned culture medium from different groups was subjected to ELISA for the indicated proteins. Values were normalized to VSMC protein concentration and plotted as the means \pm SD. (E) Carotid grafts were harvested 1 week postoperatively, before macrophage infiltration (not shown), and frozen sections were stained for the indicated protein and DNA. The ratio of immunofluorescence/DNA fluorescence in KIf15 ${ }^{-/-}$arteries was normalized to that in WT arteries and plotted as the means \pm SEM of four independent specimens of each genotype.

mouse (25) mated to Klf15flox/flox mice to generate Sm22-Cre $\mathrm{S}^{+/ 0} /$ Klf15flox/flox mutant mice (Smc-Klf15-KO) (Supplemental Figure 3A). These mice were viable and born at the expected Mendelian frequency. Assessment of KLF15 expression levels in vascular and nonvascular tissues revealed robust and specific deletion of KLF15 in aortic tissue (Supplemental Figure 3B). Smc-Klf15-KO mice were bred onto an $A p o e^{-/-}$background, and the lipid profile was assessed at 8 weeks of age. There was no significant difference between Smc-Klf15-KO/Apoe-/- and control mice with regard to total cholesterol, LDL, and triglyceride levels (Supplemental Figure 3, C-E). Moreover, systolic blood pressures assessed by tail-cuff assay were equivalent in the Smc-Klf15-KO/Apoe ${ }^{-/-}$and control mice after 15 weeks of an HFD challenge (Supplemental Figure 3F). However, after consuming an HFD for 15 weeks, Smc-Klf15-KO/Apoe - $^{-}$mice demonstrated significantly more atherosclerosis than the control mice as assessed by Sudan IV staining of aortae en face (Figure 3, A and B) and aortic transverse sections (Figure 3C and Supplemental Figure 4). Consistent with the carotid transplant studies, immunohistochemical analyses of Smc-Klf15-KO/Apoe ${ }^{-/-}$aortae showed enhanced MAC3 but reduced SMC $\alpha$-actin immunoreactivity in the aortic wall and atherosclerotic lesion, indicating more macrophages but fewer VSMCs (Figure 3C). In addition, Smc-Klf15-KO/Apoe ${ }^{-/}$aortae also showed increased MCP-1 and VCAM-1 staining in the aortic wall (Figure 3C). Collectively, these data from two distinct experimental approaches suggest that VSMC KLF15 deficiency results in enhanced vascular inflammation.

SMC-specific KLF15 deletion activates the NF- $\mathrm{K} B$ pathway. Our data show that KLF15 deficiency was associated with increased amounts of proinflammatory factors including MCP-1, VCAM-1, and MMP3, which are all established targets of NF-кB $(26,27)$. $\mathrm{NF}-\kappa \mathrm{B}$ is a principal upstream regulator of inflammatory gene expression and a well-recognized transcriptional factor involved in the pathogenesis of atherosclerosis $(26,28)$. The mammalian NF- $\mathrm{KB}$ family consists of seven proteins including p65, c-Rel, RelB, p50, p52, p100, and p105. These molecules form various homo- or heterodimers that are sequestered in the cytoplasm and translocate into the nucleus upon stimulation to activate specific downstream targets. Of note, p65 and p50 have been reported as being activated in human atherosclerosis (28-31).

As a first step, we interrogated the NF-кB pathway in nuclear extracts from aortae of Smc-Klf15-KO/Apoe-/- mice following a 

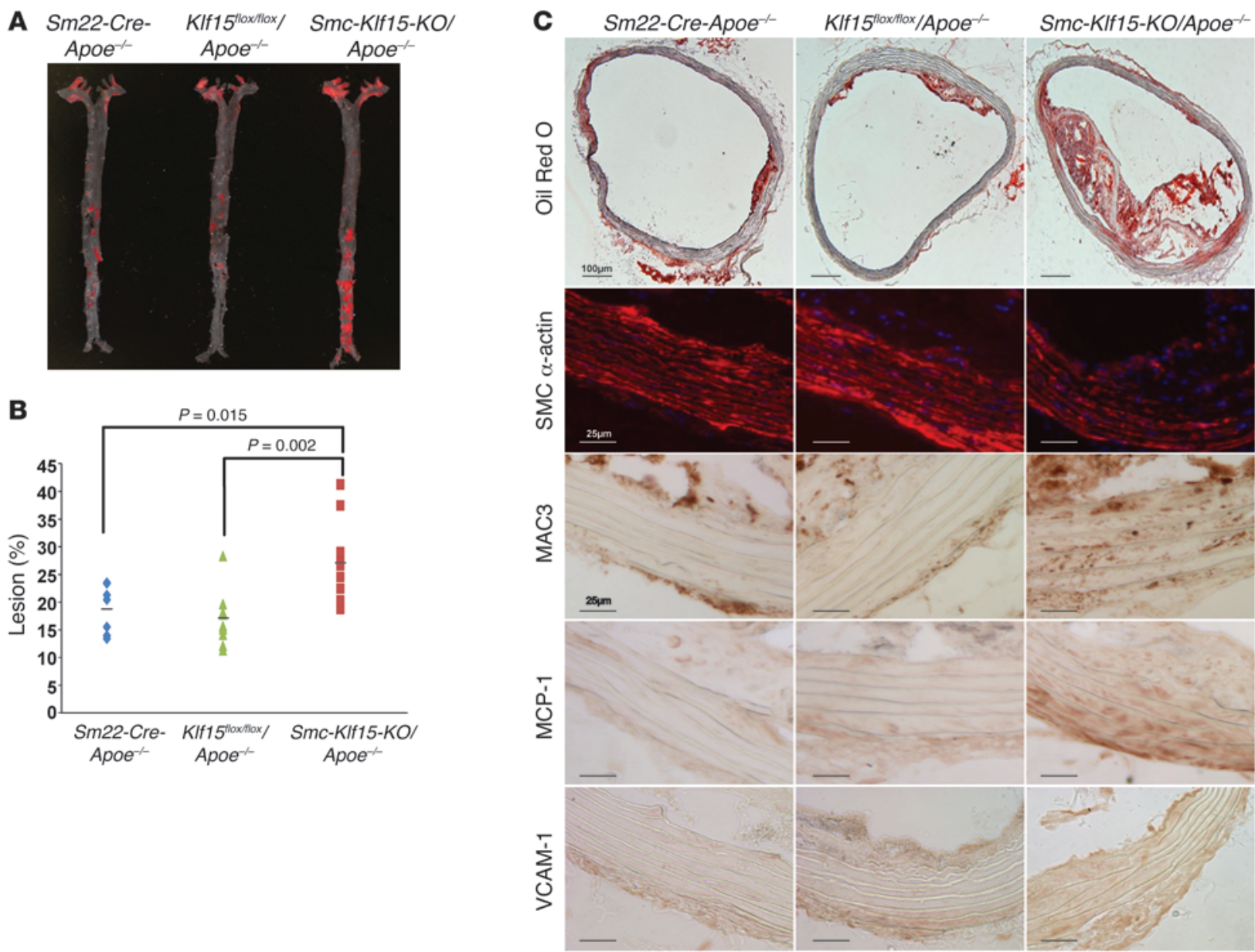

\section{Figure 3}

SMC-specific expression of KLF15 suppresses atherosclerosis. (A and B) Representative image of an Smc-Klf15-KO aorta developed substantially more atherosclerotic lesions after a 15-week HFD challenge $\left(n=7\right.$ in the Sm22Cre/Apoe ${ }^{-/}$group; $n=9$ in the KIf15 flox/flox/Apoe ${ }^{-/-}$group; and $n=11$ in the Smc-KIf15-KO/Apoe ${ }^{-/-}$group). (C) Representative immunohistochemical analyses showed enhanced atherosclerotic lesion formation as well as increased MAC3, MCP-1, and VCAM-1 staining in Smc-Klf15-KO aorta. SMC $\alpha$-actin staining was decreased in the Smc-KIf15-KO group ( $n=7$ in the Sm22Cre/Apoe ${ }^{-/-}$group; $n=9$ in the Klf15 flox/flox/Apoe ${ }^{-/-}$group; and $n=11$ in the Smc-KIf15-KO/Apoe ${ }^{-/}$group).

15-week HFD. In comparison with the control tissues, Western blot analyses revealed no significant change in the expression of total p65 and p50 (Figure 4A and Supplemental Figure 4B). Previous studies have shown that posttranslational modifications such as phosphorylation (32) or acetylation (30) can augment p65 activity. However, assessment of phosphorylated p65 (p65phosS536) revealed no significant difference between the control and KLF15-deficient aortae (Figure 4A and Supplemental Figure $4 \mathrm{~B})$. In contrast, we observed a significant increase in acetylated p65 (Ac-p65) (p65-AcK310) in SMC-KLF15-KO/Apoe-/- aortae following the 15-week HFD (Figure 4A and Supplemental Figure $4 \mathrm{~B})$. To confirm this finding, we assessed the effect on Ac-p65 (p65-AcK310) in nuclear extracts following acute knockdown of KLF15 from cultured mouse aortic VSMCs. In comparison with VSMCs treated with control virus, KLF15 knockdown engendered increased acetylation of p65 (AcK310-p65) at baseline and following TNF- $\alpha$ stimulation, but no significant change in total p65 expression (Figure 4B, right panel). Conversely, KLF15 overexpression conferred an antiparallel effect on Ac-p65 levels in human aortic SMCs (HASMCs)(Figure 4B, left panel).
Previous studies have shown that acetylation of p 65 primarily occurs in the nucleus and is regulated by histone acetyltransferases (HAT) and deacetylases. To date, seven acetylated lysines have been identified within $\mathrm{p} 65$, and the majority of these are acetylated by HAT p300/CBP (30). We therefore tested whether KLF15 altered the ability of p300 to acetylate p65 in VSMCs. We immunoprecipitated p 65 from human aortic VSMC nuclear extracts and observed that overexpression of p300 significantly increased p65 acetylation, while KLF15 overexpression repressed p300-dependent p65 acetylation (Figure 4C).

Next, we sought to explore the significance of KLF15-dependent NF- $\kappa$ B acetylation on candidate inflammatory targets such as MCP-1 and VCAM-1. To do so, we used two p65 constructs containing lysine $(\mathrm{K})$ to arginine $(\mathrm{R})$ mutations that prevent p65 acetylation: p65K7R, containing all seven lysine mutations and p65K310R, in which only Lys310 was mutated. As shown in Figure 4, D and E, in contrast to WT p65, both the p65K7R and p65K310R exhibited a reduced transactivational activity $(\sim 50 \%)$ to induce both MCP-1 and VCAM-1 promoters. The fact that similar results were seen with p65K7R and p65K310R suggests that K310 
A

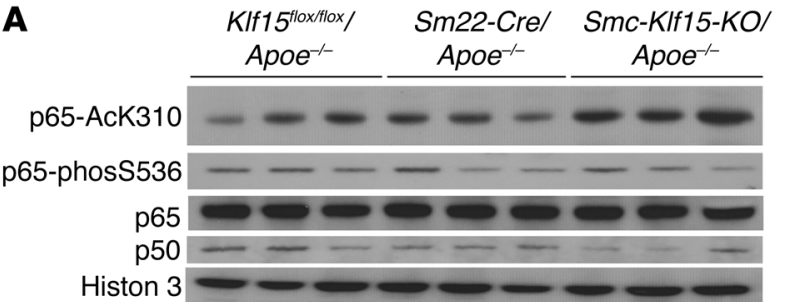

B

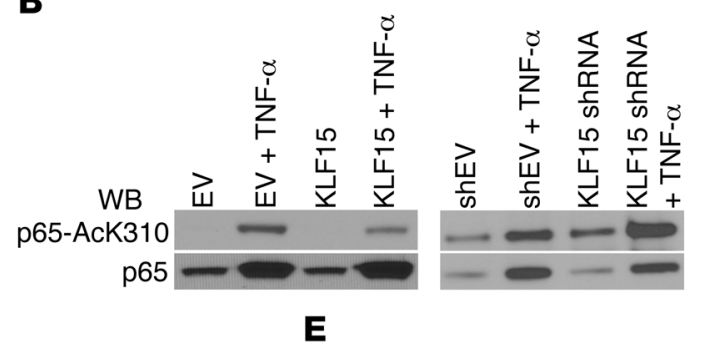

D

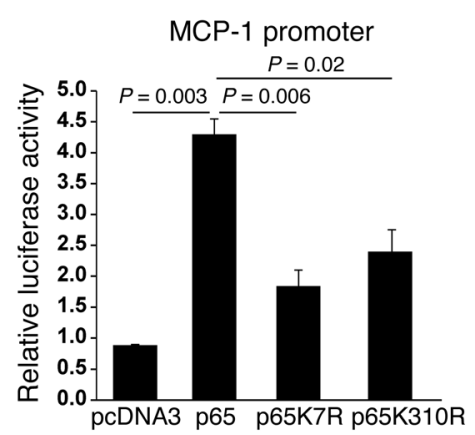

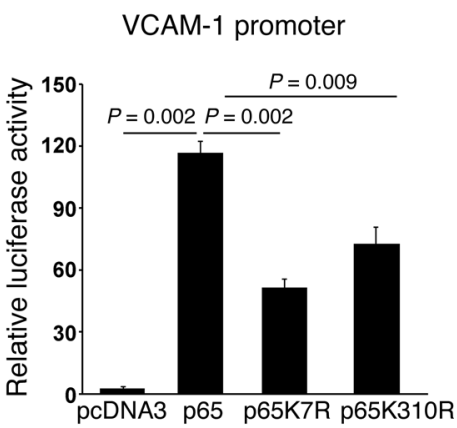

C

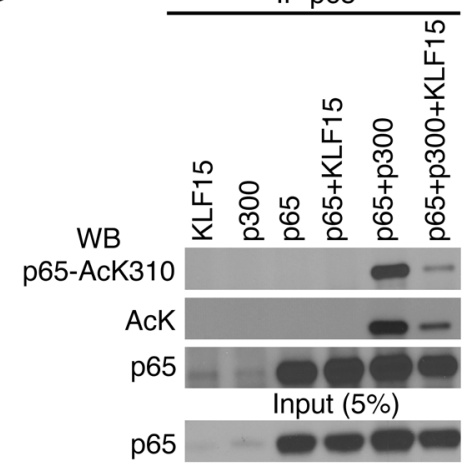

F

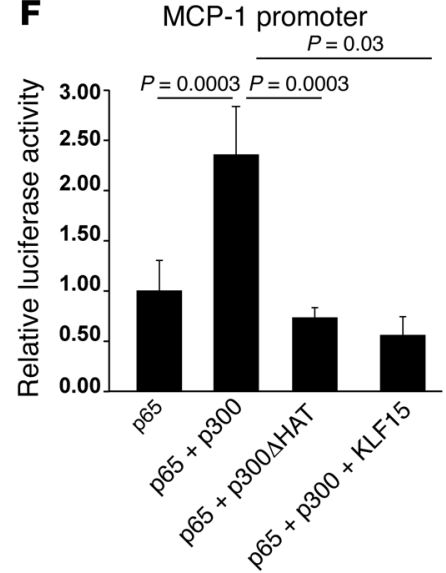

G VCAM-1 promoter

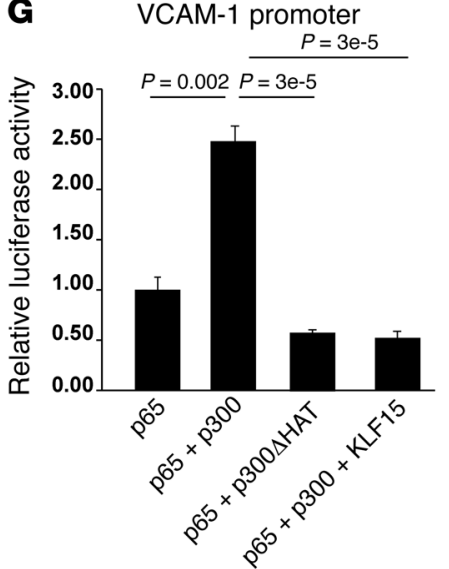

Figure 4

KLF15 suppresses NF-KB transactivation activity in VSMCs. (A) Western blots of indicated proteins in 15-week HFD-challenged aortae nuclear extract ( $n=3$ individual aortae in each group). (B) Nuclear extract Western blots of AcK310-p65 and total p65 (baseline and following TNF- $\alpha$ stimulation) in EV and KLF15 adenovirus-infected HASMCs (left panel) and in WT and KLF15 acute knockout mouse aortic SMCs. (C) HASMCs overexpressed the indicated proteins for 48 hours, then p65 was immunoprecipitated from nuclear extracts. Immunoprecipitates were immunoblotted for total p65, total acetylated lysine (AcK), and acetylated p65-lysine310 (AcK310). (D and E) HEK293T cells were transiently transfected with the indicated plasmids for 48 hours $(n=3$ independent experiments in each group). ( $\mathbf{F}$ and $\mathbf{G})$ HEK293T cells were transiently transfected by the indicated plasmids for 48 hours. p300-HAT deletion mutation showed significantly lower transcriptional activity on both MCP-1 and VCAM-1 promoters. KLF15 attenuated p300-dependent p65 activation on both promoters ( $n=3$ independent experiments in each group).

was of particular importance. To determine the effect of KLF15 on p300-augmented p65 transactivational activity, we performed cotransfection studies with p300, p65, and KLF15. As shown in Figure 4, F and G, p300 enhanced the p65-mediated activation of the MCP-1 and VCAM-1 promoters by approximately 2.5 -fold. This activation is dependent on p300 HAT activity, since a p300HAT deletion mutation (p300 $\Delta$ HAT) failed to augment p65 transcriptional activity (Figure 4, F and G). Importantly, KLF15 significantly attenuates the p300-dependent p65 activation on both the MCP-1 and VCAM-1 promoters. Collectively, the above results strongly suggest that p300-mediated acetylation is important for p65 transcriptional activity and that KLF15 attenuates this effect.
KLF15 inbibits NF- $\kappa B$ activation through KLF15-p300 interaction. We next sought to further clarify the mechanism by which KLF15 attenuates p300-dependent p65 acetylation. Since KLF factors can bind to p300 $(33,34)$, we considered the possibility that KLF15 may compete with p65 for binding to p300. Coimmunoprecipitation studies established that KLF15 can form a complex with endogenous p300 (Figure 5A). Furthermore, in a concentration-dependent manner, KLF15 overexpression inhibited the association of p300 with p65 (Figure 5B). To determine whether endogenous KLF15 affected p65-p300 interaction, we assessed the effect of KLF15 deficiency on p65-p300 interaction. In HASMCs, shRNA-mediated depletion of KLF15 increased endogenous p65-p300 interaction 
A

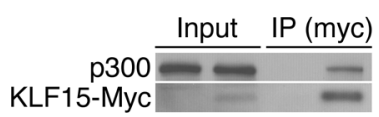

B

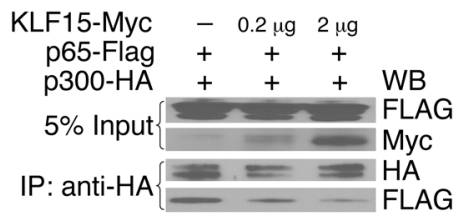

C

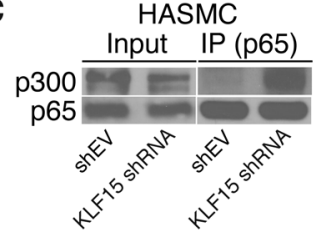

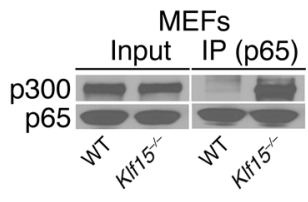

$\mathbf{F}$
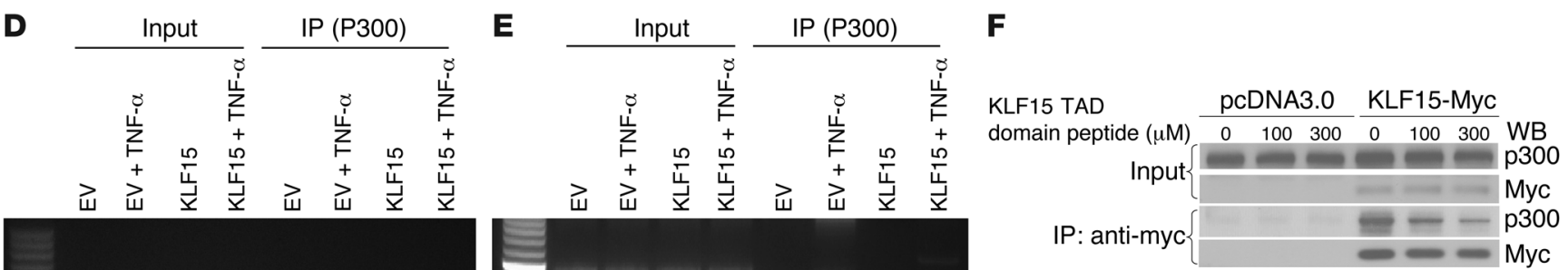

E
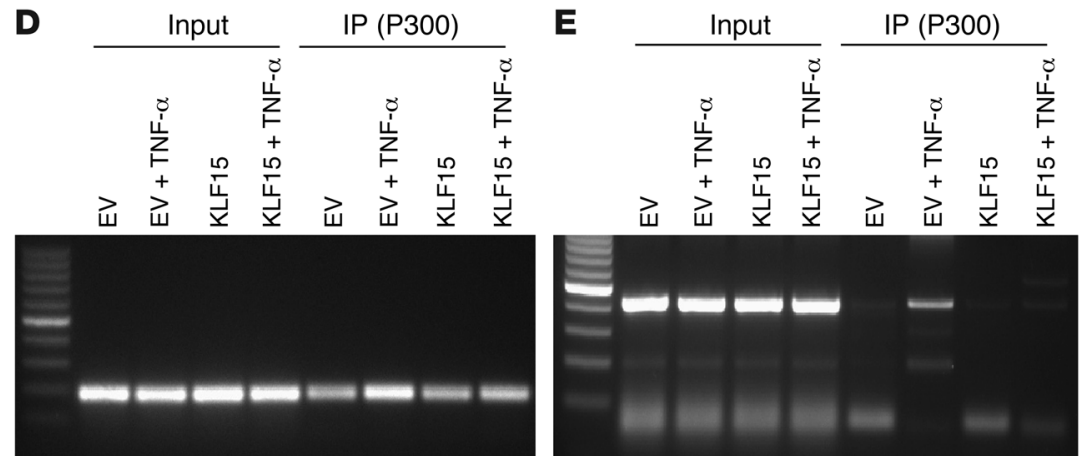

MCP-1

VCAM-1
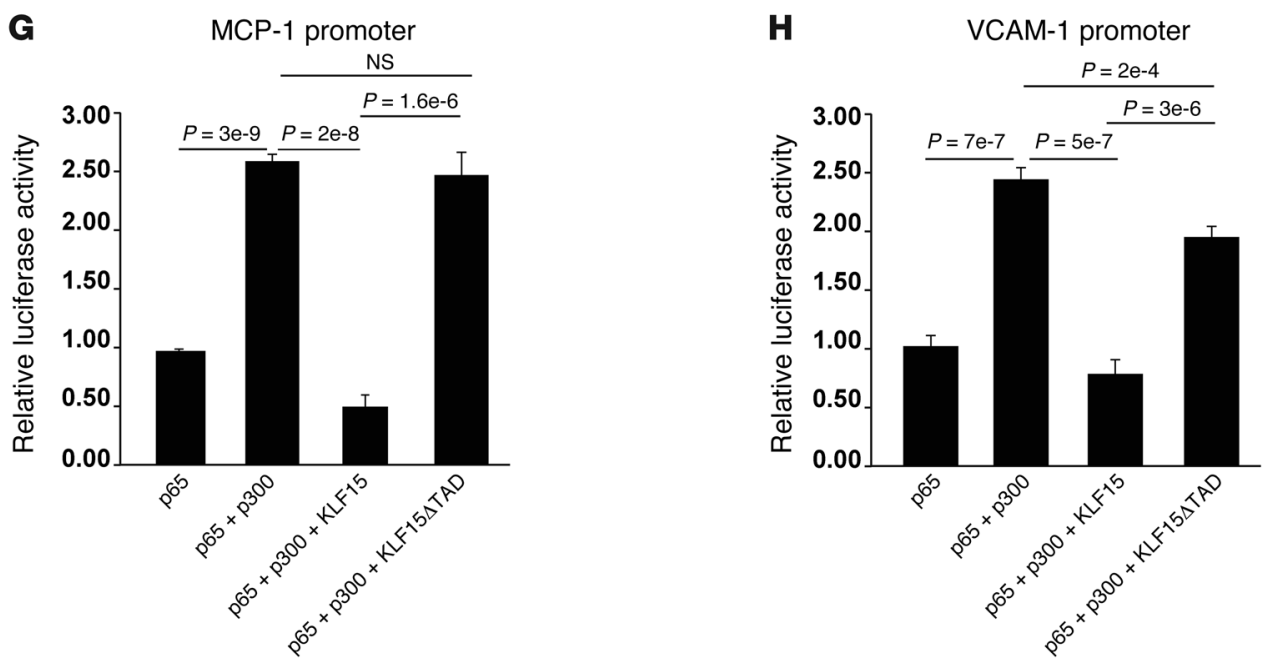

Figure 5

KLF15 inhibits NF-кB activation through KLF15-p300 interaction. (A) Coimmunoprecipitation of overexpressed myc-KLF15 and endogenous p300 in HEK293T cells. (B) KLF15 overexpression prevented p300-p65 interaction in a dose-dependent fashion in HEK293T cells. (C) Immunoprecipitation of endogenous p65 and p300 after TNF- $\alpha$ stimulation for 20 minutes in HASMCs after acute KLF15 knockdown (left panel) and in KLF15-null MEFs (right panel). (D and E) HASMCs infected with Ad-EV or Ad-KLF15 were stimulated with TNF- $\alpha$ for 4 hours. ChIPs were performed with $\mathrm{p} 300$ antibody on MCP-1 and VCAM-1 promoters containing the NF-кB binding site. (F) The 15-amino acid KLF15 TAD domain peptide competed with full-length KLF15 for p300 immunoprecipitation in HEK293T cell nuclear extracts. (G and H) Transient transfection assays showed that the KLF15 TAD domain is critical for its repressive function. KLF15 1 TAD mutant attenuated its repressive function on MCP-1 and VCAM-1 promoters ( $n=$ three independent experiments in each group).

after TNF- $\alpha$ stimulation (Figure 5C, left panel). Similar effects were also observed in KLF15-null primary mouse embryonic fibroblasts (MEFs) (Figure 5C, right panel). Next we performed ChIP assays to test whether KLF15 affected p300 occupancy on target promoters. As shown in Figure 5, D and E, KLF15 reduced p300 occupancy on the promoters of 65 targets MCP-1 and VCAM-1. Collectively, the above results suggest that KLF15 may suppress NF- $\mathrm{\kappa B}$ activity by interrupting p300-p65 interaction.

To determine the structural basis for KLF15 and p300 interaction, we took advantage of a recent finding that a specific motif within KLF1 mediates interaction with p300 (35). Since KLF15 also contains the same motif, we posited that this region may mediate interaction with $\mathrm{p} 300$. As a first step, a 15-amino acid KLF15 transactivation domain (TAD) domain peptide (VSRPFQDTLEEIEEFLEE) was synthesized for competitive immunoprecipitation assays. As shown in Figure 5F, addition of the KLF15 TAD peptide, in a dose-dependent manner, inhibited KLF15 binding to endogenous p300. Next, to determine whether the KLF15 TAD domain is functionally important, we cotransfected p65, p300, and full-length KLF15 or a KLF15 $\Delta$ TAD mutant in the presence of either an MCP-1 or VCAM-1 promoter. As shown in Figure 5, G and H, in comparison with KLF15, the ability of the KLF15 $\triangle$ TAD mutant to inhibit MCP-1 and VCAM-1 promoter activity was strongly attenuated. 


\section{Discussion}

Current paradigms for the development of vascular inflammatory disease states suggest that proinflammatory activation of the endothelium leads to immune cell recruitment and investment within the vessel wall. Subsequently, a complex interaction between immune cells and local elements such as VSMCs occurs, which contributes to disease progression $(1,7,8)$. Studies over the past decade have implicated the Kruppel-like family of factors as important regulators of inflammation (14). The current study is, we believe, the first to link KLFs to the biology of VSMCs and the development of vascular inflammation. Importantly, KLF15 was reduced in human atherosclerotic tissues (Figure 1A). Further, using two different in vivo approaches, we definitively established that VSMC KLF15 deficiency promotes VSMC inflammation and vasculopathy (Figures 2 and 3). These findings add to the growing appreciation that KLFs are central regulators of vascular inflammation. Indeed, other members of this family, such as KLF2 and KLF4, are strongly expressed in endothelial and hematopoietic lineages, perhaps owing to the common origins of these cell types (14). A feature common to both factors is their ability, in endothelial and hematopoietic cells, to promote an antiinflammatory and quiescent cellular state. Consistent with this view, endothelial deficiency of KLF4 or myeloid deficiency of KLF2 and KLF4 has been shown to promote experimental atherosclerosis $(34,36,37)$. We note that studies by Owens and colleagues support a role for KLF4 in smooth muscle biology (38). Specifically, conditional postnatal deletion of KLF4 delayed the downregulation of SMC differentiation markers but enhanced neointimal formation (39). However, the role of SMC KLF4 in a chronic inflammatory vasculitis like experimental atherogenesis remains an important unanswered question. Regardless, the current work, coupled with these previous observations, implicates KLFs as central regulators of the major cellular constituents that affect vessel homeostasis in health and disease.

An interesting aspect of the current work is the finding of a link between KLF15 and NF-KB activity. Specifically, we found that KLF15 deficiency increased NF- $\mathrm{KB}$ acetylation, an important posttranslational modification that can enhance NF- $\mathrm{KB}$ transcriptional activity. The coactivator p300 is known to interact with and acetylate p65, and thus we focused our efforts on this pathway (30). Importantly, our findings suggest that KLF15 can compete with p65 for binding to p300 in a concentration-dependent manner (Figure 5B). The importance of this interaction is further supported by the observation that depletion or deletion of KLF15 in cells enhances p65-p300 interaction (Figure 5C). Additionally, we defined the region within KLF15 that mediates this interaction and showed that deficiency of this region attenuates the ability of KLF15 to inhibit p65-p300 cooperativity (Figure 5, $\mathrm{E}-\mathrm{G})$. These findings have important implications. First, we note that previous work by our group and others has demonstrated that KLF2 and KLF4 can also interact with p300 $(34,40)$. Further, overexpression of KLF2 or KLF4 can reduce, while deficiency can augment, p300 occupancy of NF- $\mathrm{KB}$ inflammatory targets in endothelial or immune cells $(34,40,41)$. Thus, the ability to interact with p300 may serve as a shared mechanism by which KLFs in distinct cell types regulate inflammation. Second, we defined a specific 15-amino acid region within KLF15 that mediates the interaction with p300. Intriguingly, as recently pointed out by Omichinski and colleagues, this motif, which subserves p300 interaction, is common to p53 as well as to several KLFs
(KLF1, 2, 4, 5, and 15) (35). Consequently, competition between KLF1/2/4/5/15 and p53 for p300 may provide a mechanism by which these KLFs can regulate major cellular processes such as survival and adaptation to genotoxic stress. Indeed, we recently linked KLF15 deficiency to hyperacetylation of p53, enhanced VSMC apoptosis, and aneurysm formation (42). The increased p53 activity could also reduce VEGF expression (43-46), as observed in our study (Figure 2D). These observations raise the possibility that competition for p300 between KLFs and nodal regulators such as NF- $\mathrm{KB}$ and p53 may account for the broad gene regulatory and cellular effects ascribed to these factors in diverse cell types. However, technical limitations preclude the precise assessment of intrinsic concentrations and the stoichiometric relationship between these factors. Accordingly, additional studies will be required to determine whether this competition between KLF15 and specific factors (e.g., p65 or p53) for p300 is operative in vivo under various physiologic or pathologic states.

Finally, recent studies have also identified KLF15 as a central regulator of nutrient availability, flux, and energy homeostasis $(47,48)$. For example, we showed that KLF15 regulates lipid uptake and utilization in skeletal muscle (48). Whether KLF15 affects VSMC fuel utilization and energy homeostasis is unknown but is quite possible, given the intimate and evolutionarily conserved link between inflammation and metabolism across phylogeny. Since the metabolism of VSMCs is poorly understood, such efforts are likely to be highly informative and are the subject of ongoing studies.

In summary, this work identifies VSMC KLF15 as an essential regulator of vascular inflammation. These findings, coupled with recently published work in endothelial and immune cell biology, now firmly establish the KLF family members as major regulators of vessel biology (14). Further, given that the competition for coactivators is a mechanism shared by vascular KLFs, therapeutic strategies affecting this common pathway may allow for particularly robust antiinflammatory effects in multiple cell types operative in the pathogenesis of atherosclerosis and other vascular disease states.

\section{Methods}

\section{Mice and animal studies}

All mouse studies were performed in accordance with protocols approved by the IACUCs of Case Western Reserve University and Duke University.

\section{Generation and characterization of Smc-Klf15-KO mice}

Sm22-Cre transgenic mice expressing Cre recombinase under the transcriptional control of the $2.8 \mathrm{~kb}$ mouse $S m 22$ a promoter have previously been described (25). The $S m 22^{\text {Cre }}$ mouse line was a gift from Aaron Proweller's laboratory (Case Western Reserve University). The Klf15-floxed mouse line was generated by Ozgene by inserting the LoxP site flanking exon 2 of the Klf15 gene. SMC-specific Klf15 KO mice were generated by mating the Klf1 $15^{\text {flox }}$ flox mouse line with the $S m 22^{C r e}$ mouse line, designated the $\operatorname{Sm} 22-\mathrm{Cre} e^{+/ 0} / \mathrm{Klf} 15^{\text {flox/flox }}$ (Klf15-Smc-KO) mouse line. The control mice were generated from Klf15 flox/flox breeding pairs or $S m 22^{\text {Cre }}$ to WT C57BL/6J breeding pairs. To assess the expression pattern of the KlfF15 gene, Sm22-Cre $\mathrm{C}^{+/ 0} / \mathrm{Klfl} 15_{\text {flox/flox }}$ mouse aorta and liver tissues were harvested at 6 weeks of age, and Klf15 gene expression was measured by quantitative PCR (qPCR). Klf15-Smc-KO and control mouse lines were bred on an $A$ poe $e^{-/}$background for the atherosclerosis study. Klf15 $15^{--}$mice were generated as previously reported (49). Apoe-/-/ $\mathrm{Klfl}^{-/-}$mice were generated by breeding the $\mathrm{Klfl}^{-/-}$mouse line with the Apoe $e^{-/-}$mouse line. Studies were performed with age-matched controls. 


\section{Animals and diets}

Mouse atherosclerosis models were generated by feeding approximately 6- to 8-week-old male mice an atherogenic diet (Research Diet D12108-C; $1.25 \%$ cholesterol, $12.5 \%$ fat, $0 \%$ sodium cholate) for 15 or 25 weeks. All other experiments were performed with 8 - to 10 -week-old male mice with age-matched respective controls. All mouse experimentation was conducted under protocols approved by the IACUC of Case Western Reserve University.

\section{Orthotopic carotid artery transplant}

Mouse orthotopic carotid artery transplants were performed as previously described (15). C57BL/6-congenic Klf15/-- and WT mice were used as common carotid artery donors for congenic Apoe ${ }^{-/-}$mice; both donor and recipient mice were anesthetized with pentobarbital $(50 \mathrm{mg} / \mathrm{kg})$. Recipient Apoe $^{-/-}$mice were fed normal mouse chow for 1 or 4 weeks and then sacrificed. Specimens were processed for histology, and immunofluorescence was quantitated as described (15).

\section{Human subjects}

Human cDNA samples were obtained from Koichi Shimizu's laboratory at Brigham and Women's Hospital and Harvard Medical School. Briefly, nonatherosclerotic and atherosclerotic specimens were obtained from aortae from pathology specimens either from cardiac transplantation donors or autopsies ( $n=6$ each). All specimens were obtained by protocols approved by the Human Investigation Review Committee of the Brigham and Women's Hospital. As previous described, total RNA was extracted from the aortic specimens using Trizol (Invitrogen) and purified with an RNeasy kit (QIAGEN); cDNA was synthesized with Oligo(dT) and a First-Strand cDNA Synthesis Kit followed by DNase treatment (Invitrogen).

\section{Lipid profile analysis}

Total plasma cholesterol and triglyceride levels were determined after 12-hour fasting by using the respective assay kit (Cholesterol Reagent set from Pointe Scientific, Inc. and Triglyceride Reagent kit from Wako Chemicals USA). The plasma lipoprotein Western blot was performed by the University of Cincinnati Mouse Metabolic Phenotyping Center.

\section{Atherosclerosis and blood pressure analysis}

Whole-mount aortae were isolated and stained by en face Sudan IV. Descending aortae between the left and right renal arteries were cryosectioned and prepared in transverse sections for Sudan IV staining. Digital images of en face preps and transverse sections were quantitated using Image-Pro software (Media Cybernetics). Results are expressed as the percentage of the Sudan IV-positive area relative to the total aorta area. Systolic blood pressure was measured in conscious mice using the BP-2000 Blood Pressure Analysis System (Visitech Systems). To allow mice to adapt to the apparatus, we performed daily blood pressure measurements for 1 week. Blood pressure was subsequently recorded daily for 1 week.

\section{Cell culture}

Primary VSMCs, endothelial cells, macrophages, and MEFs were isolated from mice or rats as previously described $(34,41,50,51)$. Human aortic VSMCs and HEK293T cells were obtained from ATCC. All cells were cultured in DMEM supplemented with 5\% FBS. POPVC was purchased from Cayman Chemicals.

\section{Molecular biology}

Adenoviral overexpression or knockdown. Adenovirus carrying the mouse Klf15 gene (Ad-Klf15) or empty control virus (EV) was used for VSMCs. Adenovirus carrying human KLF15 shRNA or empty control virus shRNA (shEV) was used for the knockdown procedures, which were performed in HEK293T cells and HASMCs.
$R N A$ isolation and $q P C R$. Total RNA from cultured cells and mouse tissue samples was isolated using either TRIzol reagent (Invitrogen) or an RNeasy kit (QIAGEN). First-strand cDNA was synthesized and subjected to qPCR with Roche universal probe reagents (Universal ProbeLibrary; Roche Applied Science) on a StepOnePlus Real-Time PCR System (Applied Biosystems). Gene expression was normalized to $18 \mathrm{~S}$ RNA or $\beta$-actin by using the $\Delta \Delta \mathrm{Ct}$ method.

MCP-1 promoter clone. The human MCP-1 promoter covering a region from $-4,752$ to +1 (4,753 bp) was amplified from human genomic DNA by PCR and ligated into a pGL3 luciferase reporter vector (Promega). Transient transfection assays were performed with an X-tremeGENE HP transfection reagent (Roche Applied Science) in HEK293T cells. Luciferase activity in transfected cells was measured using a Luciferase Reporter Assay System (Promega). Luciferase activity was normalized to total cell protein concentration (BCA assay; Pierce Biotechnology). p65 and its mutants expression plasmids as well as p300 and its mutant expression plasmids were acquired from Addgene, deposited by Warner Green. The VCAM-1 promoter was described previously. KLF15-truncated mutant expression plasmids were clone by deletion of the following sequences: the KLF15 TAD deletion expression plasmid has a deletion of amino acids 140-160; the KLF15-ZnF-only expression plasmid has a deletion of amino acids 1-320 (accession number NP_075673.1).

Western blot analysis and immunohistochemistry. Western blots were performed following standard protocols. Antibodies were obtained as follows: p300 (Sc-585), p65 (SC-372), MCP-1 (SC-1784), VCAM-1 (SC-1504), HRPMyc (sc-40 HRP), and HRP-conjugated anti-goat IgG (SC-2020) from Santa Cruz Biotechnology Inc.; Mac-3 (550292) from BD Pharmingen; histone 3 (4499), acetylated lysine (9814), and phospho-NF-кB p65 (Ser536) (3033) from Cell Signaling Technology; goat anti-KLF4 (AF3158) from R\&D Systems; acetyl-NF-кB p65 (K310) (19870) from Abcam; p50 (06-886) and HRP-conjugated anti-rabbit light chain (MAB201P) from EMD Millipore; HA (PA1-29751) antibody from Thermo Fisher Scientific; HRP-Flag (A8592) and $\alpha$-smooth muscle actin Cy3 $3^{\mathrm{TM}}$ (C6198) from Sigma-Aldrich; and HRP-conjugated anti-rabbit IgG (NA934V) and anti-mouse IgG (NA931V) from GE Healthcare. Immunohistochemical studies of tissue samples were performed using paraffin-embedded or cryoembedded tissues. Images were collected and morphometric analysis and measurements were conducted by using Image-Pro Analyzer software (Media Cybernetics).

ELISA. Primary mouse aortic VSMCs were serum starved for 24 hours and then cultured in DMEM with no serum for 6 hours. Supernatants were collected and subjected to ELISA by Search Light (Pierce Biotechnology) for the indicated targets as shown in Figure 2D. Values were normalized to the VSMC protein concentration from the same culture dish. Experiments were performed on three independent isolated VSMC lines, each from a pool of three mice.

Immunoprecipitation. Cultured HEK293T cells, HASMCs, and MEFs were used for immunoprecipitation. Cultured HEK293T cells were transfected with KLF15-myc, p65-Flag, and/or p300-HA constructs using FuGENE HD (Promega). Nuclear protein was extracted using the NE-PER Nuclear and Cytoplasmic Extraction Reagent (Pierce Biotechnology), and anti-c-myc agarose affinity gel antibody (Sigma-Aldrich) or anti-HA agarose antibody was used for immunoprecipitation. Cultured HASMCs and MEFs were treated by human or mouse TNF- $\alpha(10 \mathrm{ng} / \mathrm{ml})$, respectively, for 20 minutes, and nuclear protein was extracted. p65 antibody (8242; Cell Signaling Technology) and magnetic Dynabeads (Invitrogen) were used for immunoprecipitation.

ChIP. ChIP assays were performed as previously described (41). In brief, $2 \times 10^{7}$ cells were stimulated with TNF- $\alpha(10 \mathrm{ng} / \mathrm{ml})$ or vehicle for 4 hours prior to cross-linking for 10 minutes with $1 \%$ formaldehyde. p300 antibody was used for ChIP. Magna ChIP Protein A Magnetic Beads and the ChIP Assay Kit were obtained from Millipore. MCP-1 promoter PCR was performed with the specific primers flanking the NF- $\kappa \mathrm{B}$ binding site (sense: 
GCCTAACCCAGGCTTGTGCCG; antisense: GGAGGTCAGTGCTGGCGTGAG), and VCAM-1 promoter PCR was performed with specific primers flanking the NF- $\mathrm{KB}-$ binding site (sense: CCCACCCCCTTAACCCACATTG; antisense: ACAGCCTGTGGTGCTGCAAGT).

\section{Statistics}

Results from gene expression in human samples (Figure 1A) and mouse carotid artery transplants (Figure $2 \mathrm{~B}$ ) are presented as the mean $\pm \mathrm{SEM}$; all other results are presented as the mean $\pm \mathrm{SD}$. Statistical significance of differences was analyzed using a two-tailed Student's $t$-test between two groups, and Bonferroni's correction was used when more than two groups were present. Mouse carotid artery data for more than two groups were analyzed by ANOVA with Tukey's post-hoc test for multiple comparisons. A $P$ value less than 0.05 was considered statistically significant.

\section{Acknowledgments}

We thank Richard Morton (Department of Cell Biology, Lerner Research Institute, Cleveland, Ohio, USA) for his assistance with the lipid profile FPLC analysis; Aaron Proweller (Case Western Reserve University) for providing the SM22-Cre mouse line; and Patrick Tso (Mouse Metabolic Phenotype Center, University of Cincinnati, Cincinnati, Ohio, USA) for his assistance with plasma lipoprotein electrophoresis. This work was supported by AHA grant 12SDG12050558 (to Y. Lu); NIH grants HL112486 (to M.K. Jain) and HL77185 (to N.J. Freedman); and an HL57506 MERIT Award (to D.I. Simon).

Received for publication December 27, 2012, and accepted in revised form June 28, 2013.

Address correspondence to: Yuan Lu, Case Western Reserve University, School of Medicine, 10900 Euclid Ave., Cleveland, Ohio 44106, USA. Phone: 216.368.0591; Fax: 216.368.0556; E-mail: yuan.lu@case. edu. Or to: Mukesh Jain, Case Western Reserve University, School of Medicine, 10900 Euclid Ave., Cleveland, Ohio 44106, USA. Phone: 216.368.3607; Fax: 216.368.0556; E-mail: mukesh.jain2@case.edu.
1. Ross R. Atherosclerosis - an inflammatory disease. NEngl J Med. 1999;340(2):115-126.

2. Virdis A, Schiffrin EL. Vascular inflammation: a role in vascular disease in hypertension? Curr Opin Nephrol Hypertens. 2003;12(2):181-187.

3. Inoue T, Croce K, Morooka T, Sakuma M, Node $\mathrm{K}$, Simon DI. Vascular inflammation and repair: implications for re-endothelialization, restenosis, and stent thrombosis. JACC Cardiovasc Interv. 2011; 4(10):1057-1066.

4. Nordon IM, Hinchliffe RJ, Loftus IM, Thompson MM. Pathophysiology and epidemiology of abdominal aortic aneurysms. Nat Rev Cardiol. 2011; 8(2):92-102.

5. Savoia C, Schiffrin EL. Inflammation in hypertension. Curr Opin Nephrol Hypertens. 2006;15(2):152-158.

6. Sullivan GW, Sarembock IJ, Linden J. The role of inflammation in vascular diseases. J Lenkoc Biol. 2000;67(5):591-602.

7. Libby P, Ridker PM, Maseri A. Inflammation and atherosclerosis. Circulation. 2002;105(9):1135-1143.

8. Wong BW, Meredith A, Lin D, McManus BM. The biological role of inflammation in atherosclerosis. Can J Cardiol. 2012;28(6):631-641.

9. Verma S, Gupta M, Ridker PM. Therapeutic targeting of inflammation in atherosclerosis: we are getting closer. Can J Cardiol. 2012;28(6):619-622.

10. Charo IF, Taub R. Anti-inflammatory therapeutics for the treatment of atherosclerosis. Nat Rev Drug Discov. 2011;10(5):365-376.

11. Mitchell RN, Libby P. Vascular remodeling in transplant vasculopathy. Circ Res. 2007;100(7):967-978.

12. Raines EW. PDGF and cardiovascular disease. Cytokine Growth Factor Rev. 2004;15(4):237-254.

13. Feinberg MW, Lin Z, Fisch S, Jain MK. An emerging role for Kruppel-like factors in vascular biology. Trends Cardiovasc Med. 2004;14(6):241-246.

14. Alaiti MA, Orasanu G, Tugal D, Lu Y, Jain MK. Kruppel-like factors and vascular inflammation: implications for atherosclerosis. Curr Atheroscler Rep. 2012;14(5):438-449.

15. Zhang L, et al. Expression of tumor necrosis factor receptor- 1 in arterial wall cells promotes atherosclerosis. Arterioscler Thromb Vasc Biol. 2007; 27(5):1087-1094

16. Ketelhuth DF, Back M. The role of matrix metalloproteinases in atherothrombosis. Curr Atheroscler Rep. 2011;13(2):162-169.

17. von der Thusen JH, Kuiper J, van Berkel TJ, Biessen EA. Interleukins in atherosclerosis: molecular pathways and therapeutic potential. Pharmacol Rev. 2003;55(1):133-166.

18. Dixon WG, Symmons DP. What effects might anti-TNFalpha treatment be expected to have on cardiovascular morbidity and mortality in rheumatoid arthritis? A review of the role of TNF $\alpha$ in cardiovascular pathophysiology. Ann Rheum Dis. 2007;66(9):1132-1136.

19. Cugno M, Ingegnoli F, Gualtierotti R, Fantini F Potential effect of anti-tumour necrosis factor-alpha treatment on reducing the cardiovascular risk related to rheumatoid arthritis. Curr Vasc Pharmacol. 2010;8(2):285-292.

20. Emanuele E, et al. Association of plasma eotaxin levels with the presence and extent of angiographic coronary artery disease. Atherosclerosis. 2006; 186(1):140-145.

21. Gross MD, et al. Circulating soluble intercellular adhesion molecule 1 and subclinical atherosclerosis: the Coronary Artery Risk Development in Young Adults Study. Clin Chem. 2012;58(2):411-420.

22. Tang W, et al. Association of SICAM-1 and MCP-1 with coronary artery calcification in families enriched for coronary heart disease or hypertension: the NHLBI Family Heart Study. BMC Cardiovasc Disord. 2007;7:30

23. Jones KL, Maguire JJ, Davenport AP. Chemokine receptor CCR5: from AIDS to atherosclerosis. $\mathrm{BrJ}$ Pharmacol. 2011;162(7):1453-1469.

24. Cybulsky MI, et al. A major role for VCAM-1, but not ICAM-1, in early atherosclerosis. J Clin Invest. 2001; 107(10):1255-1262.

25. Lepore JJ, Cheng L, Min Lu M, Mericko PA, Morrisey EE, Parmacek MS. High-efficiency somatic mutagenesis in smooth muscle cells and cardiac myocytes in SM22alpha-Cre transgenic mice. Genesis. 2005;41(4):179-184.

26. Brand K, Page S, Walli AK, Neumeier D, Baeuerle PA. Role of nuclear factor-kappa B in atherogenesis. Exp Physiol. 1997;82(2):297-304.

27. Bond M, Chase AJ, Baker AH, Newby AC. Inhibition of transcription factor NF-kappaB reduces matrix metalloproteinase- $1,-3$, and -9 production by vascular smooth muscle cells. Cardiovasc Res. 2001; 50(3):556-565.

28. Monaco C, et al. Canonical pathway of nuclear factor kappa B activation selectively regulates proinflammatory and prothrombotic responses in human atherosclerosis. Proc Natl Acad SciUS A. 2004; 101(15):5634-5639.

29. Ruland J. Return to homeostasis: downregulation of NF-KB responses. Nat Immunol. 2011;12(8):709-714.

30. Huang B, Yang XD, Lamb A, Chen LF. Posttranslational modifications of NF-kappaB: another layer of regulation for NF-kB signaling pathway. Cell Signal. 2010;22(9):1282-1290.

31. Chen LF, Greene WC. Regulation of distinct biological activities of the NF-kappaB transcription fac- tor complex by acetylation. J Mol Med (Berl). 2003; 81(9):549-557.

32. Yang F, Tang E, Guan K, Wang CY. IKK beta plays an essential role in the phosphorylation of RelA/ p 65 on serine 536 induced by lipopolysaccharide. J Immunol. 2003;170(11):5630-5635.

33. Urvalek AM, Wang X, Lu H, Zhao J. KLF8 recruits the p300 and PCAF co-activators to its amino terminal activation domain to activate transcription. Cell Cycle. 2010;9(3):601-611.

34. Zhou G, et al. Endothelial Kruppel-like factor 4 protects against atherothrombosis in mice. J Clin Invest. 2012;122(12):4727-4731.

35. Mas C, et al. Structural and functional characterization of an atypical activation domain in erythroid Kruppel-like factor (EKLF). Proc Natl Acad Sci U S A. 2011;108(26):10484-10489.

36. Lingrel JB, et al. Myeloid-specific Kruppel-like factor 2 inactivation increases macrophage and neutrophil adhesion and promotes atherosclerosis. Circ Res. 2012;110(10):1294-1302.

37. Sharma N, et al. Myeloid Krüppel-like factor 4 deficiency augments atherogenesis in $\mathrm{ApoE}^{-/-}$mice - brief report. Arterioscler Thromb Vasc Biol. 2012; 32(12):2836-2838.

38. Liu Y, Sinha S, McDonald OG, Shang Y, Hoofnagle $\mathrm{MH}$, Owens GK. Kruppel-like factor 4 abrogates myocardin-induced activation of smooth muscle gene expression. J Biol Chem. 2005; 280(10):9719-9727.

39. Yoshida T, Kaestner KH, Owens GK. Conditional deletion of Kruppel-like factor 4 delays downregulation of smooth muscle cell differentiation markers but accelerates neointimal formation following vascular injury. Circ Res. 2008;102(12):1548-1557.

40. Mahabeleshwar GH, et al. The myeloid transcription factor KLF2 regulates the host response to polymicrobial infection and endotoxic shock. Immunity. 2011;34(5):715-728.

41. Liao X, et al. Kruppel-like factor 4 regulates macrophage polarization. J Clin Invest. 2011; 121(7):2736-2749.

42. Haldar SM, et al. Klf15 deficiency is a molecular link between heart failure and aortic aneurysm formation. Sci Transl Med. 2010;2(26):26ra26.

43. Mukhopadhyay D, Tsiokas L, Sukhatme VP. Wildtype p53 and v-Src exert opposing influences on human vascular endothelial growth factor gene expression. Cancer Res. 1995;55(24):6161-6165.

44. Zhang L, et al. Wild-type p53 suppresses angiogenesis in human leiomyosarcoma and synovial sarcoma by transcriptional suppression of vascular endothelial growth factor expression. Cancer Res. 2000;60(13):3655-3661. 
45. Pal S, Datta K, Mukhopadhyay D. Central role of p53 on regulation of vascular permeability factor/ vascular endothelial growth factor (VPF/VEGF) expression in mammary carcinoma. Cancer Res. 2001;61(18):6952-6957.

46. Qin G, et al. Cell cycle regulator E2F1 modulates angiogenesis via p53-dependent transcriptional control of VEGF. Proc Natl Acad Sci U S A. 2006;
103(29):11015-11020.

47. Jeyaraj D, et al. Klf15 orchestrates circadian nitrogen homeostasis. Cell Metab. 2012;15(3):311-323.

48. Haldar SM, et al. Kruppel-like factor 15 regulates skeletal muscle lipid flux and exercise adaptation. Proc Natl Acad Sci U S A. 2012;109(17):6739-6744.

49. Gray S, et al. The Kruppel-like factor KLF15 regulates the insulin-sensitive glucose transporter
GLUT4. J Biol Chem. 2002;277(37):34322-34328.

50. Lu Y, et al. Kruppel-like factor 15 regulates smooth muscle response to vascular injury--brief report. Arterioscler Thromb Vasc Biol. 2010;30(8):1550-1552.

51. Kawanami D, et al. Kruppel-like factor 2 inhibits hypoxia-inducible factor 1 alpha expression and function in the endothelium. J Biol Chem. 2009; 284(31):20522-20530. 